\title{
Increased Cul1 expression promotes melanoma cell proliferation through regulating p27 expression
}

\author{
GUANGDI CHEN and GANG LI \\ Department of Dermatology and Skin Science, Jack Bell Research Centre, Vancouver Coastal Health \\ Research Institute, University of British Columbia, Vancouver, British Columbia, Canada
}

Received June 16, 2010; Accepted July 20, 2010

DOI: 10.3892/ijo_00000786

\begin{abstract}
Cullin1 (Cul1) serves as a rigid scaffold in SCF (Skp1/Cullin/Rbx1/F-box protein) complex, the largest family of ubiquitin-protein E3 ligases, and aberrant expression of Cul1 is involved in dysfunction of SCF E3 ligases. Previously, we found that Cul1 expression is increased in early stages of melanoma. In the present study, we further investigated the role of Cul1 in melanoma development. Our results showed that knockdown of Cul1 inhibits melanoma cell growth while overexpression of Cull enhances cell proliferation through the control of cell cycle progression. We also found that Cul1 regulates melanoma cell growth and cell cycle progression through degradation of $\mathrm{p} 27$ by functional SCF Skp2 $^{\text {complex. }}$ This study elucidates the role of Cul1 in melanoma cell proliferation and improves our understanding of increased expression of Cul1 in early stages of melanoma.
\end{abstract}

\section{Introduction}

The cutaneous malignant melanoma derived from epidermal melanocytes is the most serious type of skin cancer. The incidence of melanoma increases steadily in the order of 3-7\% per year for fair-skinned Caucasian populations (1). In 1935, the lifetime risk for an American developing invasive melanoma was 1 in 1500. In 2007, this risk is 1 in 63 for invasive melanomas and 1 in 33 if in situ melanomas were included (2-4). With surgical excision, the early diagnosed melanomas are curable, however, up to $20 \%$ of patients will develop metastatic tumors due to its high capability of invasion and rapid metastasis to other organs $(5,6)$. The prognosis for patients with advanced melanoma remains poor, and the 5-year survival rate for patients with distant metastases is less than $10 \%$ (7).

Cancer development is the process by which normal cells are transformed into cancerous cells, which results

Correspondence to: Dr Gang Li, Jack Bell Research Centre, 2660 Oak Street, Vancouver, BC V6H 3Z6, Canada

E-mail: gangli@interchange.ubc.ca

Key words: Cul1, melanoma, cell proliferation, cell cycle from aberrant cellular responses to stimuli. This process is regulated by transcription, translation, post-translational modifications and degradation of key regulatory proteins which has a crucial role in maintaining and regulating cellular homeostasis $(8,9)$. The ubiquitin-proteasome system controls the abundance of a number of cellular proteins, including p53, ß-catenin, p21, p27 and cyclins (10-12). Polyubiquitin conjugation of lysine residues in proteins as a result of collaboration with a ubiquitin-activating enzyme (E1), a ubiquitin-conjugation enzyme (E2), and a ubiquitin ligase (E3) is required for selective recognition and degradation by the $26 \mathrm{~S}$ proteasome (13). Dysfunction of E3 ubiquitin ligases contributes to abnormal cell growth and differentiation (12). The SCF (Skp1/Cullin/Rbx1/F-box protein) complexes are the largest family of Cullin RING ligases (CRL) and ubiquitinate a broad range of proteins $(14,15)$. As a rigid scaffold in SCF complex, Cullin1 (Cul1) plays a key role in SCF complex assembly and aberrant expression of Cul1 results in dysfunction of SCF E3 ligases. Loss of Cul1 results in early embryonic lethality and deregulation of cyclin $\mathrm{E}$ (16). Cul1 is modified through the covalent attachment of the ubiquitin-like small molecule Nedd8 and an intact NEDD8 pathway is required for Cullin-dependent ubiquitylation in mammalian cells (17). It has been reported that c-Myc activates the expression of Cul1 gene and promotes ubiquitindependent proteolysis and cell cycle progression (18). Previously, we have shown that Cul1 expression is increased in early stages of melanoma (19). In this study, we further investigated the role of Cull in melanoma cell proliferation.

\section{Materials and methods}

Cell culture. The melanoma cell lines, MMRU and PMWK, were obtained as previously described (20) and were maintained in DMEM supplemented with 10\% FBS (Invitrogen, Burlington, Ontario, Canada). The cells were cultured in a $5 \% \mathrm{CO}_{2}$ atmosphere at $37^{\circ} \mathrm{C}$.

Plasmids, siRNA and transfection. HA-Cul1 and Myc-Skp2 plasmids were provided by Dr M. Pagano at New York University and Dr J. Hsieh from Washington University at St. Louis, respectively. Plasmids were transfected into cells using the Effectene reagent (Qiagen, Mississauga, Ontario, Canada). The Cul1 siRNA oligomers were synthesized by Dharmacon (Lafayette, CO). The control scramble siRNA 
A

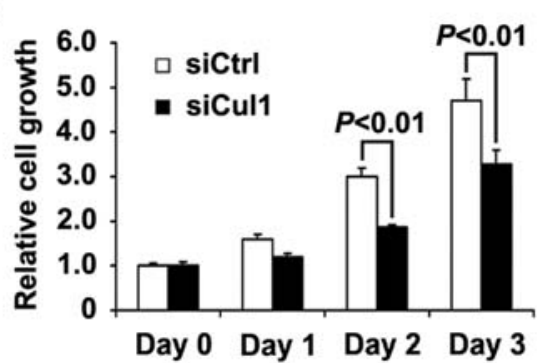

B

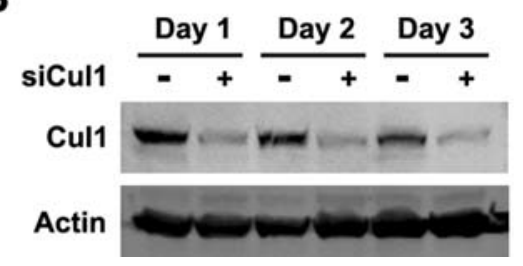

C

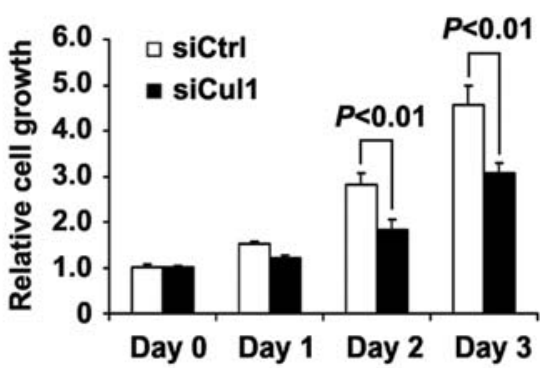

D

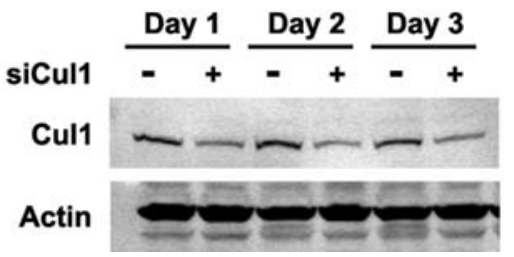

Figure 1. Knockdown of Cul1 inhibits melanoma cell growth. (A) MMRU cells were transfected with Cul1 siRNA and the cell growth was examined by SRB cell proliferation assay. (B) Cullexpression in MMRU cells from (A) was examined by Western blot analysis. (C) PMWK cells were transfected with Cul1 siRNA and the cell growth was examined by SRB cell proliferation assay. (D) Cull expression in PMWK cells from (C) was examined by Western blot analysis.

and Skp2 siRNA oligomers were obtained from Qiagen. siRNA was transfected into cultured cells using the SilentFect reagent (Bio-Rad, Mississauga, Ontario, Canada).

Western blot analysis. Cellular proteins were resolved on $12 \%$ SDS-PAGE and electrotransfered onto polyvinylidene difluoride membranes (Bio-Rad). Membranes were blocked and then subsequently incubated with primary antibodies and secondary antibodies labelled with the fluorescent dyes IRDye 800 or IRDye 680 (LI-COR Biosciences, Lincoln, $\mathrm{NE}$ ), followed by protein visualization on the Odyssey Infrared Imaging System (LI-COR Biosciences). The primary antibodies included rabbit anti-cyclin D1 and cyclin E, mouse anti-p21, p27, Cul1, Skp2, (Santa Cruz Biotechnology, Santa Cruz, CA), HA, myc (Epitope Biotech, Vancouver, BC, Canada), and actin (Sigma, St. Louis, MO).

SRB cell proliferation assay. Cells were transfected with HACul1 plasmid or Cull siRNA. Twenty-four hours after transfection, $2 \times 10^{4}$ cells were seeded in each well of 24-well plates, and then the SRB cell proliferation assay was performed as previously described (21).

Flow cytometry. The cells were treated with $1 \mu \mathrm{g} / \mathrm{ml}$ aphidicolin for overnight and then released in fresh medium for 0 and $6 \mathrm{~h}$. Then cells were collected and fixed with $70 \%$ ethanol at $4^{\circ} \mathrm{C}$ for overnight, stained with $40 \mu \mathrm{g} / \mathrm{ml}$ propidium iodide (PI) in hypotonic fluorochrome buffer $(0.1 \%$ Triton $\mathrm{X}-100$, $0.1 \%$ sodium citrate, and $25 \mu \mathrm{g} / \mathrm{ml}$ RNase A) for $30 \mathrm{~min}$. Samples were then analyzed using a FACSCanto flow cytometer (BD Biosciences).

Statistical analysis. For SRB cell proliferation assays and flow cytometry analysis, Student's t-test was used. Data represent means \pm SD from three independent experiments. A $\mathrm{P}<0.05$ was considered significant.

\section{Results}

Cull enhances cell proliferation in melanoma cells. Since Cul1 expression was increased in melanoma when comparing with dysplastic nevi (19), we hypothesized that Cull might affect melanoma cell growth. The MMRU cells were transfected with siRNA targeting Cul1 to knock down Cul1 expression and the cell growth was analyzed by SRB assay. The results showed that knockdown of Cul1 dramatically inhibited MMRU cell growth (Fig. 1A and B). Similarly, knockdown of Cul1 inhibited cell growth in another melanoma cell line, PMWK (Fig. 1C and D). On the other hand, MMRU cells were transfected with HA tagged Cul1 plasmids to overexpress Cull and we found that overexpression of Cul1 enhanced cell growth (Fig. 2A and B). The similar effect of overexpression of Cull enhancing cell growth was found in PMWK cells (Fig. 2C and D). These data indicate that Cull can regulate melanoma cell growth.

Cull regulates G1-S phase transition in cell cycle progression. We next examined if knockdown of Cul1 induced inhibition on melanoma cell growth is due to cell apoptosis. The MMRU cells were transfected with Cull siRNA and collected for cell population analysis by fluorescence activate cell sorting (FACS). The results showed that Cul1 knockdown did not increase the sub-G1 cell population, which was designated as apoptotic cells. Cul1 knockdown did not enhance UV-induced cell apoptosis either (Fig. 3A). Thus, we hypothesized that Cul1 may regulate cell growth through the control of cell cycle progression. MMRU cells were transfected with Cul1 siRNA and treated with aphidicolin to synchrorize the cells at G1 phase. The cells were released in fresh medium without aphidicolin to progress and the cell cycle progression was analyzed by FACS analysis. The results showed that Cul1 knockdown arrested cells at G1 phase by inhibiting G1 to $\mathrm{S}$ phase transition (Fig. 3B and C). On the other hand, we 
A

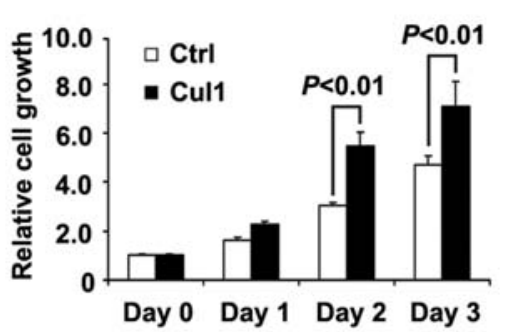

B

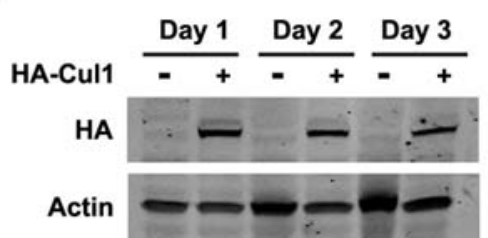

C
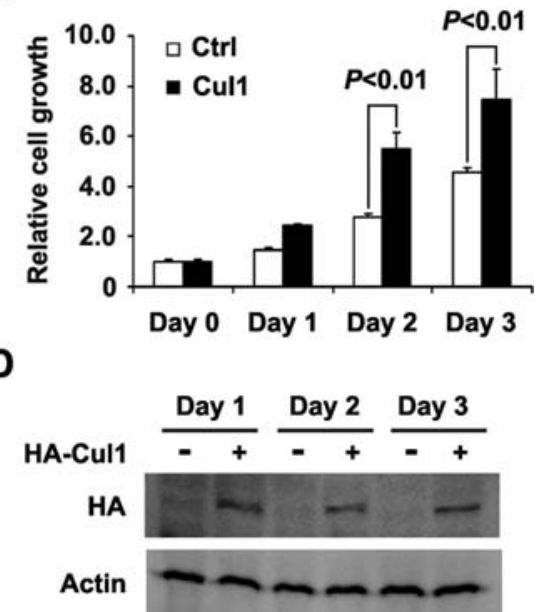

Figure 2. Overexpression of Cul1 promotes melanoma cell growth. (A) MMRU cells were transfected with HA-Cul1 plasmids to overexpress Cul1 and the cell growth was examined by SRB assay. (B) Overexpression of HA-Cul1 in the MMRU cells from (A) was examined by Western blot analysis. (C) PMWK cells were transfected with HA-Cull plasmids to overexpress Cull and the cell growth was examined by SRB assay. (D) Overexpression of HA-Cul1 in the PMWK cells from (C) was examined by Western blot analysis.

A

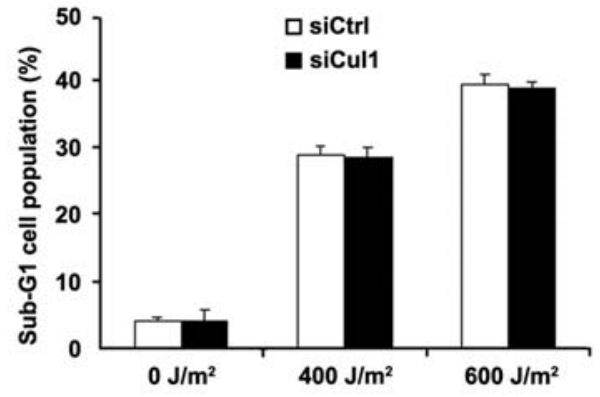

B

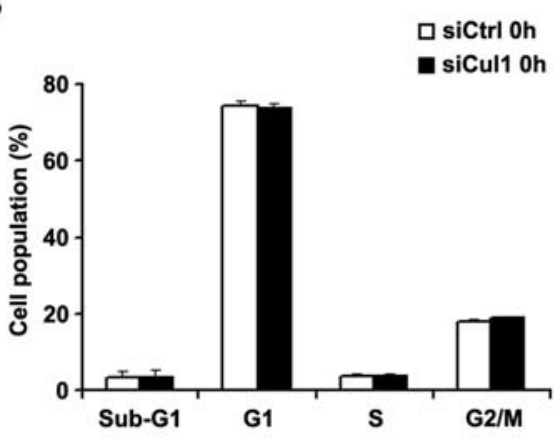

D

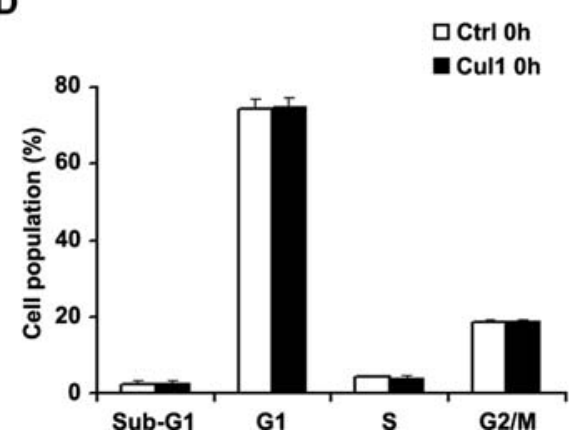

C

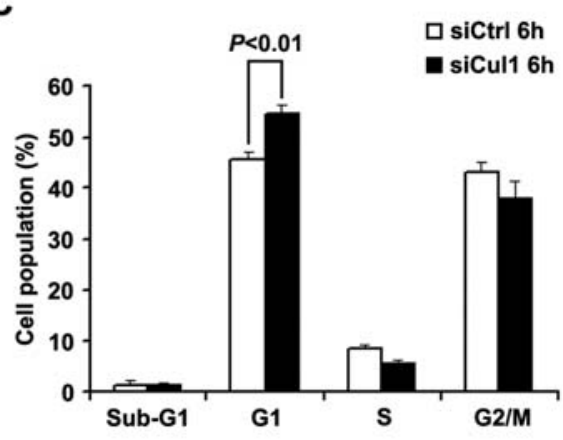

$\mathbf{E}$

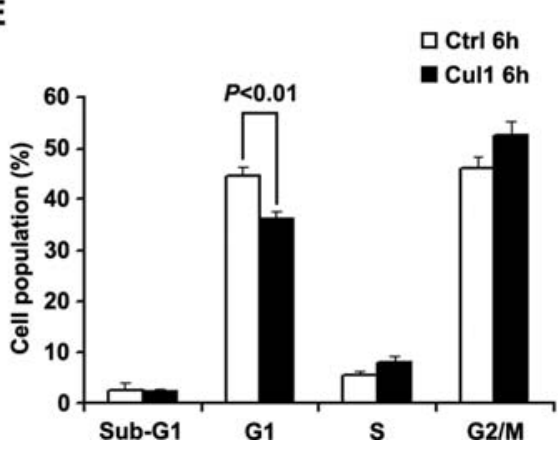

Figure 3. Cul1 regulates G1 to S phase transition during cell cycle progression. (A) MMRU cells were transfected with Cul1 siRNA for $24 \mathrm{~h}$ and irradiated with UVB as indicated. Twenty-four hours after UVB irradiation, the cells were collected and cell population was examined by FACS analysis. (B and C) MMRU cells were transfected with Cul1 siRNA and treated with $1 \mu \mathrm{g} / \mathrm{ml}$ aphidicolin overnight. The cells were released in fresh medium for 0 (B) or $6 \mathrm{~h}(\mathrm{C})$ and cell cycle progression was examined by FACS analysis. (D and E) MMRU cells were transfected with HA-Cul1 plasmids and treated with $1 \mu \mathrm{g} / \mathrm{ml}$ aphidicolin overnight. The cells were released in fresh medium for 0 (D) or $6 \mathrm{~h}$ (E) and cell cycle progression was examined by FACS analysis. 

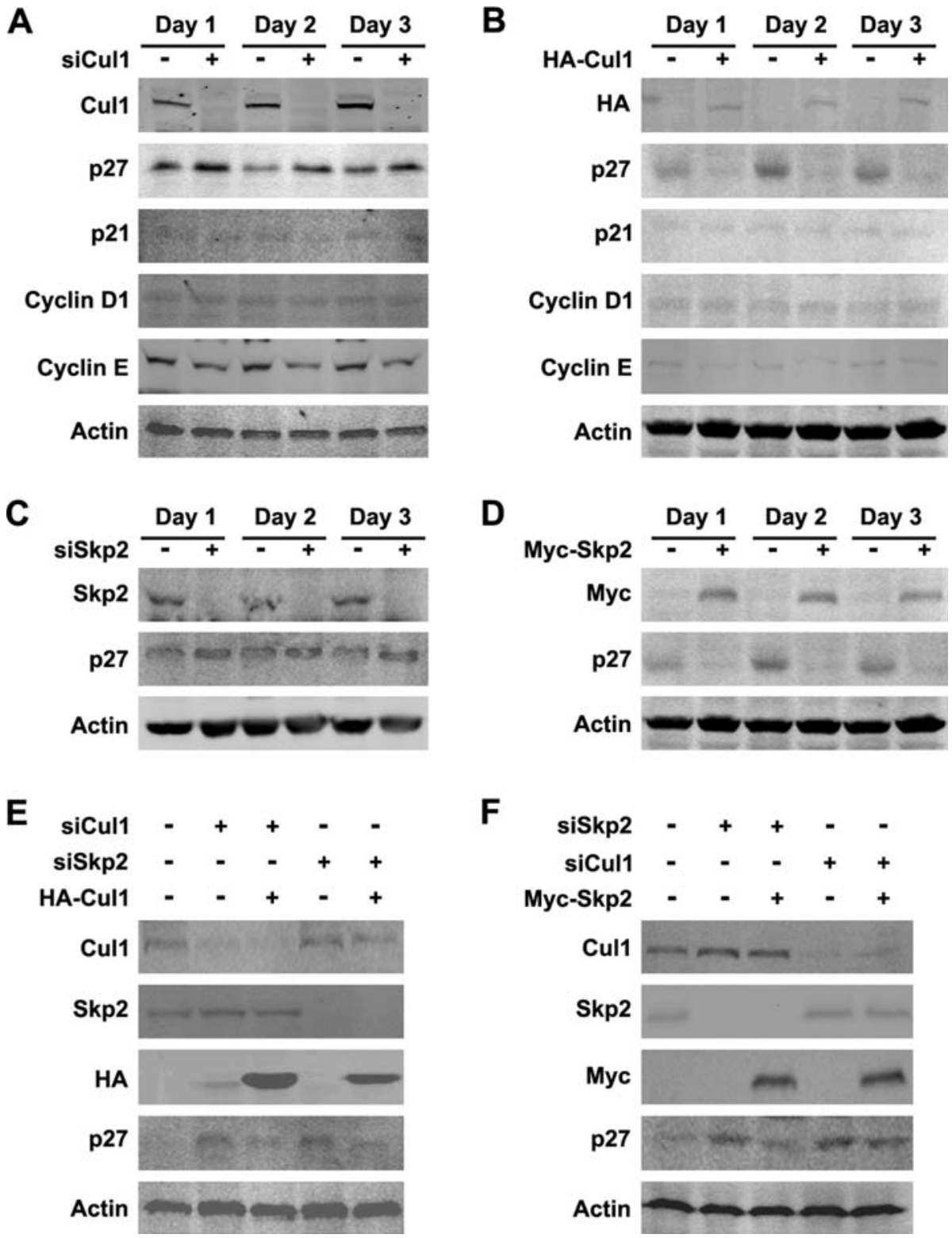

Figure 4. Cul1 regulates p27 expression through functional SCF ${ }^{\text {Skp2 }}$ complex. (A and B) MMRU cells were transfected with Cul1 siRNA (A) or HA-Cul1 plasmids (B) and the expression of Cul1, p27, p21, Cyclin D1 and Cyclin E was examined by Western blot analysis. (C and D) MMRU cells were transfected with Skp2 siRNA (C) or Myc-Skp2 plasmid (D) and the expression of Skp2, p27 and actin was examined by Western blot analysis. (E and F) MMRU cells were transfected with Cul1 or Skp2 siRNA for $24 \mathrm{~h}$ followed by transfection with HA-Cul1 (E) or Myc-Skp2 plasmids (F). The expression of Cul1, Skp2, p27 and actin was examined by Western blot analysis.

found that overexpression of Cul1 promoted cell cycle progression through enhancing G1 to S phase transition (Fig. 3D and E). Therefore, Cul1 regulates melanoma cell growth through regulating cell cycle progression.

Cull regulates $p 27$ expression through functional $S C F^{5 k p 2}$ complex. We next examined the mechanism of Cul1 regulation on cell cycle progression. Cell cycle progression is strictly controlled by cyclins, cyclin-dependent kinases (Cdks), and Cdk inhibitors $(22,23)$. Since cyclin D1, cyclin E, and Cdk inhibitors p27 and p21 play key roles in G1 to S phase transition during cell cycle progression $(24,25)$, we examined the expression of these cyclins and Cdk inhibitors in Cul1 knockdown cells. The results showed that Cul1 knockdown increased p27 expression in the MMRU cells but did not affect the expression of p21, cyclin D1 and cyclin E (Fig. 4A). Conversely, overexpression of Cul1 decreased p27 expression in the MMRU cells but not the expression of p21, cyclin D1 and cyclin E (Fig. 4B). As it was shown that Skp2 SCF E3 ligase targets p27 for its degradation $(26,27)$, we next examine if the F-box protein Skp2 regulates p27 expression. We found that knock-down of Skp2 increased p27 expression while overexpression of Skp2 decreased p27 expression (Fig. 4C and D). These data prompt us to hypothesize that Cull may cooperate with Skp2 to regulate p27 expression. We found that overexpression of Cul1 attenuated Skp2 knockdownmediated increase of p27 expression. Similarly, overexpression of Skp2 decreased p27 expression in Cul1 knockdown cells (Fig. 4E and F). These results suggest that Cul1 regulates p27 expression through functional SCFSkp2 complex. 


\section{Discussion}

Cell proliferation is completed by cell cycle progression, which is regulated by cyclins, Cdks and Cdk inhibitors $(22,23)$. We found that the ability of cell proliferation was drastically decreased after Cul1 knockdown in melanoma cells, which is due to inhibition of cell cycle progression by arresting cell cycle at G1 phase, but not apoptosis. Our data indicated that Cul1 cooperates with Skp2 in SCF complex to degrade p27 and promote cell cycle progression. Gain of Skp2 and loss of p27 protein expression are implicated in melanoma progression where the level of p 27 may be regulated by targeted proteolysis via Skp2 $(28,29)$. Expression of Skp2 and 27 proteins exhibited a significant inverse relationship and significantly correlated with increasing American Joint Committee on Cancer (AJCC) tumor classification and AJCC stage (28). Moreover, vertical growth phase (VGP) melanomas show significant higher nuclear Skp2 expressions when compared with the radial growth phase (RGP) (29). However, we did not find increased expression of Cul1 in advanced melanomas when comparing primary melanomas (19). We speculate that Cul1 expression may correlate with Skp2 expression but inversely with p27 expression during melanoma progression.

In human cells, inactivation of Cul4A, another cullin protein, induces p27 stabilization and G1 cell cycle arrest $(30,31)$. It has been speculated that the F-box protein Skp2 cooperates with Cul1-Skp1 as well as Cul4A-DDB1 to induce proteolysis of p27 (32). We found that overexpression of Skp2 reduced p27 expression in Cul1 knockdown melanoma cells, suggesting that Skp2 may utilize other E3 ligase complex to degrade p27 in melanoma cells, like Cu14A based E3 ligase. We also found that overexpression of Cul1 can reduce p27 expression in Skp2 knockdown melanoma cells, indicating that there may be other Skp2-independent E3 ligases which are involved in the p27 degradation pathway. Previously, Lim et al demonstrated p27 degradative function of Skp2 in low-grade lymphomas but not in aggressive lymphomas (33), which suggests that other factors contribute to deregulation of p27 expression in these tumors. It has been reported that Wnt-induced turnover of p27 was independent from classical

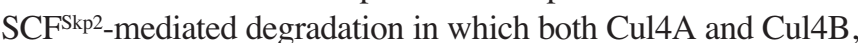
components of an alternative E3 ubiquitin ligase, were required for Wnt-induced p27 degradation and S-phase progression (34).

Since the major difficulty in treating melanoma especially for metastatic melanoma is its resistance to radio- and chemotherapy, the systemic therapy for melanoma remains unsatisfactory $(35,36)$. Although the mechanism for drug resistance in melanoma is still unclear, it appears more important to explore how melanoma develop and find biomarkers for melanoma diagnosis especially at early stage. We found that Cull expression was increased in early stage of melanomas, indicating that Cul1 may serve as a potential marker for human melanoma initiation. On the other hand, the development of pharmaceutical compounds targeting specific SCF ubiquitin ligases is timely and is complemented by basic biochemical studies that have identified substrates for important cellular regulators such as $\beta-\operatorname{TrCP}$ and Skp2 (37). In melanoma, it has been reported that knockdown of Skp2 inhibited the melanoma cell growth in vitro and suppressed tumor proliferation in vivo, suggesting that gene silencing of Skp2 can be a potent tool of cancer gene therapy for suppression of p27 degradation in malignant melanoma (38). We found that knockdown of Cul1 inhibited melanoma cell growth is partly through Skp2 targeting p27 degradation pathway. Thus, targeting Cul1 in gene therapy may hold promise for melanoma treatment at early stage.

In conclusion, Cul1 knockdown inhibits melanoma cell growth by arresting cells at G1 phase via regulating p27 expression through $\mathrm{SCF}^{\text {Skp2 }}$ complex, which improves our understanding of increased expression of Cul1 in early stages of melanoma and implies targeting Cull may serve as a potential therapy for melanoma.

\section{Acknowledgments}

We gratefully thank Drs M. Pagano and J. Hsieh for kindly providing the plasmids. This work was supported by Canadian Institutes of Health Research (MOP-93810) and Canadian Dermatology Foundation (G.L.). G.C. is a recipient of Postdoctoral Trainee Award from Michael Smith Foundation for Health Research jointly funded with VGH \& UBC Hospital Foundation.

\section{References}

1. Diepgen TL and Mahler V: The epidemiology of skin cancer. $\mathrm{Br}$ J Dermatol 146 (Suppl. 61): 1-6, 2002.

2. Rigel DS, Friedman RJ and Kopf AW: The incidence of malignant melanoma in the United States: issues as we approach the 21st century. J Am Acad Dermatol 34: 839-847, 1996.

3. Rigel DS: The effect of sunscreen on melanoma risk. Dermatol Clin 20: 601-606, 2002.

4. Rigel DS: Cutaneous ultraviolet exposure and its relationship to the development of skin cancer. J Am Acad Dermatol 58: S129-S132, 2008.

5. Balch CM, Buzaid AC, Soong SJ, et al: Final version of the American Joint Committee on Cancer staging system for cutaneous melanoma. J Clin Oncol 19: 3635-3648, 2001.

6. Houghton AN and Polsky D: Focus on melanoma. Cancer Cell 2: 275-278, 2002

7. Trinh VA: Current management of metastatic melanoma. Am J Health Syst Pharm 65: S3-S8, 2008.

8. Paul S: Dysfunction of the ubiquitin-proteasome system in multiple disease conditions: therapeutic approaches. Bioessays 30: 1172-1184, 2008.

9. Hung MS, Mao JH, Xu Z, et al: Cul4A is an oncogene in malignant pleural mesothelioma. J Cell Mol Med 23: 572-574, 2010.

10. Ciechanover A: The ubiquitin-proteasome proteolytic pathway. Cell 79: 13-21, 1994.

11. Glickman $\mathrm{MH}$ and Ciechanover $\mathrm{A}$ : The ubiquitin-proteasome proteolytic pathway: destruction for the sake of construction. Physiol Rev 82: 373-428, 2002.

12. Reinstein E and Ciechanover A: Narrative review: protein degradation and human diseases: the ubiquitin connection. Ann Intern Med 145: 676-684, 2006.

13. Schrader EK, Harstad KG and Matouschek A: Targeting proteins for degradation. Nat Chem Biol 5: 815-822, 2009.

14. Vodermaier HC: $\mathrm{APC} / \mathrm{C}$ and SCF: controlling each other and the cell cycle. Curr Biol 14: R787-R796, 2004.

15. Nakayama KI and Nakayama K: Ubiquitin ligases: cell-cycle control and cancer. Nat Rev Cancer 6: 369-381, 2006.

16. Dealy MJ, Nguyen KV, Lo J, et al: Loss of Cul1 results in early embryonic lethality and dysregulation of cyclin E. Nat Genet 23: 245-248, 1999.

17. Ohh M, Kim WY, Moslehi JJ, et al: An intact NEDD8 pathway is required for Cullin-dependent ubiquitylation in mammalian cells. EMBO Rep 3: 177-182, 2002

18. O'Hagan RC, Ohh M, David G, et al: Myc-enhanced expression of Cul1 promotes ubiquitin-dependent proteolysis and cell cycle progression. Genes Dev 14: 2185-2191, 2000. 
19. Chen G, Cheng Y, Martinka M and Li G: Cul1 expression is increased in early stages of human melanoma. Pigment Cell Melanoma Res (In press).

20. Chen G, Wang Y, Garate M, Zhou J and Li G: The tumor suppressor ING3 is degraded by $\mathrm{SCF}^{\text {Skp2 }}$-mediated ubiquitinproteasome system. Oncogene 29: 1498-1508, 2010.

21. Li G, Tang L, Zhou X, Tron V and Ho V: Chemotherapy-induced apoptosis in melanoma cells is p53-dependent. Melanoma Res 8: 17-23, 1998.

22. Reed SI: Ratchets and clocks: the cell cycle, ubiquitylation and protein turnover. Nat Rev Mol Cell Biol 4: 855-864, 2003.

23. Hochegger H, Takeda S and Hunt T: Cyclin-dependent kinases and cell-cycle transitions: does one fit all? Nat Rev Mol Cell Biol 9: 910-916, 2008

24. Lee MH and Yang HY: Regulators of G1 cyclin-dependent kinases and cancers. Cancer Metastasis Rev 22: 435-449, 2003.

25. Li W, Sanki A, Karim RZ, et al: The role of cell cycle regulatory proteins in the pathogenesis of melanoma. Pathology 38: 287-301, 2006.

26. Carrano AC, Eytan E, Hershko A and Pagano M: SKP2 is required for ubiquitin-mediated degradation of the CDK inhibitor p27. Nat Cell Biol 1: 193-199, 1999.

27. Tsvetkov LM, Yeh KH, Lee SJ, Sun H and Zhang H: p27(Kip1) ubiquitination and degradation is regulated by the $\mathrm{SCF}(\mathrm{Skp} 2)$ complex through phosphorylated Thr187 in p27. Curr Biol 9: 661-664, 1999.

28. Li Q, Murphy M, Ross J, Sheehan C and Carlson JA: Skp2 and $\mathrm{p} 27^{\mathrm{kip} 1}$ expression in melanocytic nevi and melanoma: an inverse relationship. J Cutan Pathol 31: 633-642, 2004.

29. Woenckhaus C, Maile S, Uffmann S, et al: Expression of Skp2 and $\mathrm{p} 27^{\mathrm{KIP} 1}$ in naevi and malignant melanoma of the skin and its relation to clinical outcome. Histol Histopathol 20: 501-508, 2005.
30. Higa LA, Yang X, Zheng J, et al: Involvement of CUL4 ubiquitin E3 ligases in regulating CDK inhibitors Dacapo/p2 $7^{\text {Kip1 }}$ and cyclin E degradation. Cell Cycle 5: 71-77, 2006.

31. Li B, Jia N, Kapur R and Chun KT: Cul4A targets p27 for degradation and regulates proliferation, cell cycle exit, and differentiation during erythropoiesis. Blood 107: 4291-4299, 2006.

32. Bondar T, Kalinina A, Khair L, et al: Cul4A and DDB1 associate with $\mathrm{Skp} 2$ to target $\mathrm{p} 27^{\mathrm{Kip} 1}$ for proteolysis involving the COP9 signalosome. Mol Cell Biol 26: 2531-2539, 2006.

33. Lim MS, Adamson A, Lin Z, et al: Expression of Skp2, a p27(Kip1) ubiquitin ligase, in malignant lymphoma: correlation with p27(Kip1) and proliferation index. Blood 100: 2950-2956, 2002.

34. Miranda-Carboni GA, Krum SA, Yee K, et al: A functional link between Wnt signaling and SKP2-independent p27 turnover in mammary tumors. Genes Dev 22: 3121-3134, 2008.

35. Li Y and McClay EF: Systemic chemotherapy for the treatment of metastatic melanoma. Semin Oncol 29: 413-426, 2002.

36. Soengas MS and Lowe SW: Apoptosis and melanoma chemoresistance. Oncogene 22: 3138-3151, 2003.

37. Frescas D and Pagano M: Deregulated proteolysis by the F-box proteins SKP2 and beta-TrCP: tipping the scales of cancer. Nat Rev Cancer 8: 438-449, 2008.

38. Katagiri Y, Hozumi Y and Kondo S: Knockdown of Skp2 by siRNA inhibits melanoma cell growth in vitro and in vivo. J Dermatol Sci 42: 215-224, 2006. 